\title{
Classification of Female Apparel using Convolutional Neural Network
}

\author{
Qiao-Qi Li ${ }^{\text {a }}$, Yue-Qi Zhong ${ }^{\mathrm{a}, \mathrm{b}, *}, \quad$ Xin Wang ${ }^{\mathrm{a}}$ \\ ${ }^{a}$ Donghua University, Shanghai 201620, China \\ ${ }^{\mathrm{b}}$ Key Lab of Textile Science and Technology, Ministry of Education, China
}

\begin{abstract}
With the vigorous development of clothing e-commerce, the amount of clothing image data on the internet has increased dramatically. A tedious effort was required to manually label and classify the semantic attributes of clothing images. Manual marking is time-consuming and laborious, so a method of automatic classification using convolutional neural networks was studied. In this paper, a female cloth dataset consisting of 10 types of female clothing was built. Convolutional Neural Network (CNN) was employed to learn the feature vectors for each type. Five different types of architectures, including ResNet50, Inception-v3, and VGG-19, AlexNet, and FashionNet were used for performance comparison. Experimental results have shown that Inception-v3 possesses the highest accuracy $(98.07 \%$ for training and $96.91 \%$ for testing) in clothing classification compared with other methods.
\end{abstract}

Keywords: Female Clothing Image; Image Classification; Convolutional Neural Network; Deep Learning

\section{Introduction}

There is a rapid growth of e-commerce. Apparel online retail is a large part of that which account for about $25 \%$ of overall Internet transactions [1]. This phenomenon has led to a huge number of online fashion products data. Correctly labeling these data can facilitate applications such as similar clothing retrieval and relevant products recommendation. The rapid development of the e-commerce industry has brought convenience to our lives, but at the same time, it has put forward new and higher requirements for traditional search: identifying and classifying objects in pictures, and searching, one of the more extensive problems is the identification of clothing types. The traditional recognition and classification technology rely on manual or digital image processing, pattern recognition, through image detection and segmentation, feature extraction and classification recognition, etc. The disadvantage is that the extracted features are mostly characterized by the specified features, and can not describe all the images well. The properties of objects in the middle, and often require large calculations, etc., are time-consuming and laborintensive, and are not suitable for calculation on portable devices.

${ }^{*}$ Corresponding author.

Email address: zhyq@dhu.edu.cn (Yue-Qi Zhong). 
Currently, in major e-commerce shopping platform, the method to label fashion products mostly requires prior fine classification of clothing images and affix the corresponding label. At present, most of the methods of labeling are manual labeling. In the environment where the number of garment images is exploding, the method of manual labeling has many drawbacks, and there are several serious disadvantages as follows: 1) Keywords can only describe abstract features that are easy to extract and abstract. It can not fully reflect the visual characteristics of clothing images; 2), People need accurate keyword text annotation for each image. However, the number of images is huge and requires a great deal of manpower and resources for manual annotation; 3) Due to the subjectivity of human cognition, different people may have different understandings about the same image. When image keyword text is annotated, subjectivity and uncertainty, thus affecting the classification results. By contrast, clothing classification based on image content can save lots of human labor and also give more objective results. The final goal of this project is to be able to auto label or score each product based on one or more unconstrained clothing images. In this work, the experimental target was focused on female apparel images.

Various algorithms have been proposed to solve image-based clothing classification problem. The current research progress is as follows: Alem [2] classified clothing into 23 categories based on attitude estimation and color, SIFT and other features. Bourdev [3] developed a system to describe the appearance of people. In addition, for garment segmentation, $\mathrm{Hu}$ [4] proposed the use of foreground and background estimates based on the Constrained Delaunay Triangulation (CDT), which does not require any pre-defined garment models. Weber [5] and others proposed the use of attitude detectors to solve the problem of clothing occlusion. Manfredi [6] proposed a clothing segmentation algorithm based on popular store dataset. There were also some researchers dedicated to the classification of clothing attributes, such as color, collar, sleeves or other attributes. Chen [7] introduced a fully automatic system that produces garments with a list of named properties. Lorenzo-Navarro J [8] evaluated the LBP and HOG descriptors' ability to classify clothing attributes. The aforementioned work used handcraft features to address the classification problem.

Deep Learning is an evolving technology and also is able to solve apparel image classification efficiently. In recent years, the feature extraction method based on deep learning has received great attention and has a good application effect in natural image classification. It can effectively solve the problem of clothing image classification, and minimize the abstraction process on data features. Reduce human intervention. In 2012, Krizhevsky Alex et al. [9] used CNN to win the ImageNet Image Recognition Contest and took the second place. At present, CNN has achieved remarkable results in various image processing tasks. For example, the Deep ID model proposed by Sun et al. [10] had achieved more than 99\% accuracy in face recognition. Simonyan et al. [11] proposed two-stream CNN that combines two factors of time and space for motion recognition and got a good result. VGG-NET [12] achieved first and second place respectively in 2014 ILSVRC positioning and classification. GoogLeNet [13] won the ILSVRC Classification Challenge in 2014 with a top 5 error rate of $6.67 \%$. GoogLeNet proposed a new model component named "Inception Module", which is a NIN (Network In Network) structure. Simply by increasing the number of layers did not further improve network performance, and sometimes will be counterproductive. To solve this problem, Microsoft Research Institute proposed Deep Residual Network (Residual Network) in 2015 [14]. ResNet increased the network layers to 152. At this time, the training process of the whole network could be normalized and achieved a better performance.

Lao et al [15] trained Convolutional Neural Network and obtained $50.2 \%$ and $74.5 \%$ accuracy respectively in the types of clothing and attribute classification. Bao [1] adopted a Convolu- 
tional Neural Network with metric learning to classify clothing images based on the attributes of clothing. The accuracy of classification results was between $48 \%$ and $78 \%$. Li [16] and others explored the clothing classification retrieval model based on the clothing attributes. They used Convolutional Neural Network to automatically learn the characteristics of different attributes of clothing. Then, they achieved an efficient classification of the clothing attributes. Simultaneously they extracted 160 dimensions and SIFT features from the training, and they built a hash coding. Then established a hash index to achieve fast clothing image retrieval. The final experimental results showed accuracy rate between $90 \%$ and 95\%. Rohit et al [17] used Convolutional Neural Network to classify clothing images, but the results were not satisfactory. The training accuracy based on clothing style never exceeded $55 \%$, while the accuracy based on clothing attributes was less than $70 \%$. In this research, we employ trained deep convolutional neural networks to classify clothing types because it outperforms conventional machine learning algorithms in the object detection problem [18, 19], and finally achieves $96 \%$ accuracy. rate.

Clothing image classification based on Convolutional Neural Network may not generate high precisely when there are complex images in the background or the image quality is poor. In this paper, we demonstrate the comparisons among different architectures with a proposed small dataset.

\section{Convolutional Neural Network (CNN)}

In deep learning, convolutional neural network is a deep feedforward artificial neural network, which has been successfully applied to image recognition. CNN is usually composed of multiple convolution layers, pooling layers, and full connection layers. Each layer can transform the representation from a lower level to a slightly higher level. With enough transformation, a raw input signal can be translated into high-level features which have good abstract representation. The effect of each layer can be defined as:

$$
z^{(i+1)}=w^{(i)} x^{(i)}+b^{(i)}
$$

The output is:

$$
x^{(i+1)}=f\left(z^{(i)}\right)
$$

where $w^{(i)}$ is the i-th weights, $b^{(i)}$ is the bias. The activation function used in this paper was ReLU function, which could effectively solve the problem of gradient disappearance with accelerated convergence.

The role of the pooling layer is to reduce the dimension. The full connection layer for the output is usually defined as the last layer(s) connected to the output layer for final prediction. In our practice, we chose Softmax function as the last layer of a classifier.

\section{Experiments and Discussion}

\subsection{Create a Dataset: Female Cloth Dataset}

Because of the huge amount of model parameters, insufficient data size will lead to serious overfitting, which means poor generalization. Therefore, a female cloth dataset with 15000 images was 
constructed. The data set contained 10 categories: labe, suit, shirt, windbreaker, jacket, shirt, sportswear, T-shirt, underwear, socks, hat. All the female images in this dataset were downloaded from open-source websites. The backgrounds, sizes and lighting conditions of these images were unconstrained. The dataset was split into the training set and test set with a ratio of 3:2, 9000 randomly selected samples were used as training set, and 6000 were used as test set. The amount of training and testing samples for each label was shown in Table 1. Each type of image had 600 sheets as a test set. Table 1 showed the types of images in the database and the amount of training and test samples for each label.

Table 1: Female clothing image training and test sample quantity distribution

\begin{tabular}{ccccccccccc}
\hline label & suit & shirt & windbreaker & jacket & kirt & sportswear & T-shirt & underwear & socks & hat \\
\hline training & 900 & 900 & 900 & 900 & 900 & 900 & 900 & 900 & 900 & 900 \\
testing & 600 & 600 & 600 & 600 & 600 & 600 & 600 & 600 & 600 & 600 \\
total & 1500 & 1500 & 1500 & 1500 & 1500 & 1500 & 1500 & 1500 & 1500 & 1500 \\
\hline
\end{tabular}

Figure 1 illustrated several examples of the female cloth dataset. The background, lighting conditions and other factors were not considered when selected images. The image size will be adjusted to $227 * 227$ in subsequent work.

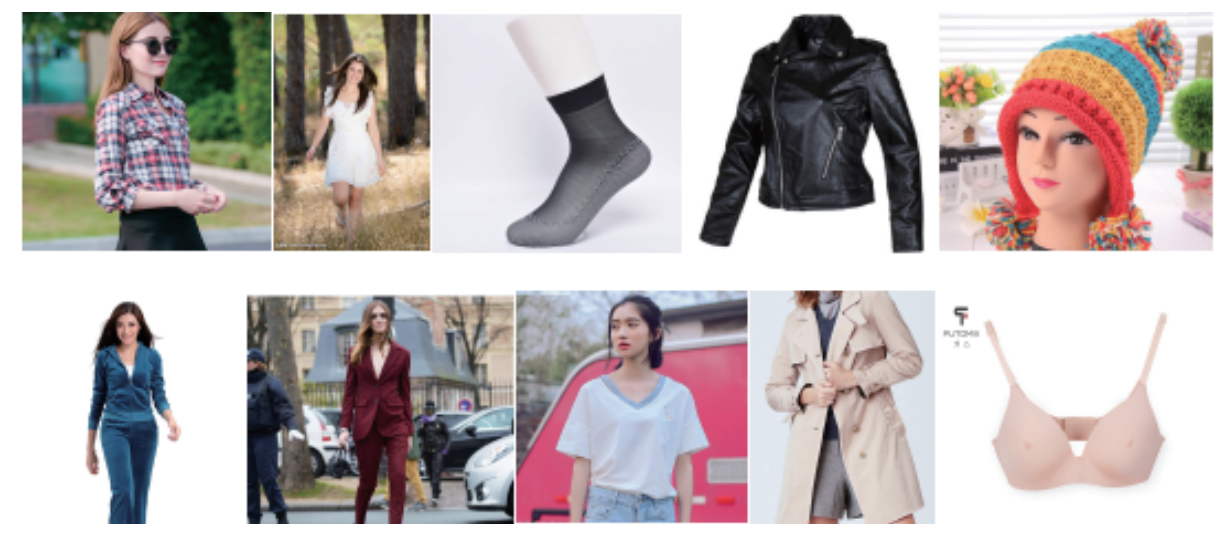

Fig. 1: Examples of data

\subsection{Female Image Classification}

The workflow in this study included image preprocessing, network training and training parameter tuning. The overall process was as shown in Figure 2. Preprocessing was mainly to change the image to a standard image that meets the input requirements of CNN. Including the image resizing, image flipping (Included left and right flipping and flipping up and down, etc.), unified image size adjusted to $227 \times 227$. The goal of image preprocessing was to increase the order of magnitude and reduce the amount of computation. This can save a lot of training time. After preprocessing, processed data was iteratively fed into the model.

During backpropagation, the parameters were updated using the gradient descent. Through multiple forward and back propagation, constantly updated convolution layers, fully connection layers and other parameters. Then made the network gradually close to the optimal solution. 


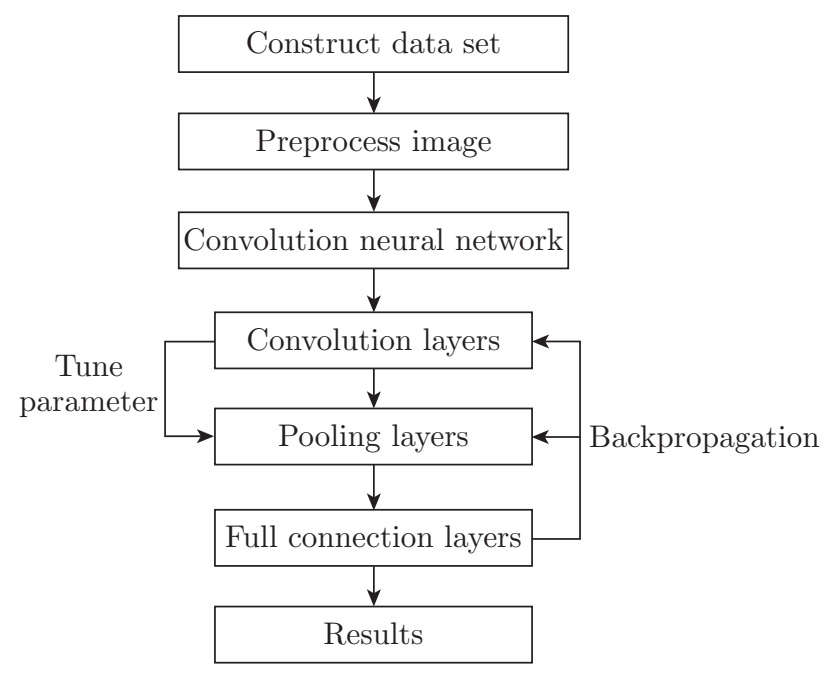

Fig. 2: Schematic diagram of the training

Convolutional Neural Network architecture used in this work includes ResNet-v1-50, Inceptionv3, VGG 19, AlexNet, FashionNet. VGG-19 consists of 16 convolutional and 3 fully-connected layers. In each convolutional layers, small convolution filters of size $3 \times 3$ are used to extract local features and reduce the number of parameters. Inception-v3 is one of GoogleNet series which is famous for its Inception module. Inception module allows for parallel convolution and pooling operations benefits from its "Network in Network" architecture. Traditional fully-connected layers are replaced by average pooling to save memory. ResNet-v1-50 is an efficient network because of its identity connection between input and output of each Residual module, which helps for fitting very deep convolutional network.

All Convolutional Neural Network models were implemented using TensorFlow [20]. Experimental training process lasted 16h with computing equipment of GTX 1060 GPU, 3GB memory. During training, learning rate was initialized to 0.01, and decayed with exponential decay factor 0.93 after every 1000 epoch. The momentum was set to 0.9999 , and the weight attenuation coefficient was set to 0.00004 . The experiment set a total of 160000 iterations of training.

\subsection{Results and Discussion}

The experimental results of three CNN models on the female cloth dataset were shown in Table 2. The accuracy of the classification results of the three models on the female cloth dataset was all high. The effect of inception-v3 was relatively good, with an accuracy rate of $96.91 \%$ and a resnet-v1-50 rate of 93.54\%. VGG 19 accuracy of $94.97 \%$. AlexNet accuracy of $87.26 \%$. And FashionNet accuracy of $88.58 \%$. Experimental results showed that CNN has obvious advantages in image classification, higher accuracy than other methods. Among these three models, Inceptionv3 is the best performer with the highest accuracy.

Because the Inception-v3 is the best performing network with the highest classification accuracy. Inception-v3 could increase the depth and width of the network while reducing the number of parameters. Therefore, it could effectively solve the problem of over-fitting, and accelerate the calculation. The extra computing power used to deepen the network and increase the network nonlinearity. Therefore, the classification accuracy compared with the other two CNN models had 
Table 2: Clothing type classification results

\begin{tabular}{cccccc}
\hline & Resnet-v1-50 & Inception-v3 & VGG 19 & AlexNet & FashionNet \\
\hline Training set & $94.23 \%$ & $98.07 \%$ & $96.68 \%$ & $89.34 \%$ & $91.37 \%$ \\
Test set & $93.54 \%$ & $96.91 \%$ & $94.97 \%$ & $87.26 \%$ & $88.58 \%$ \\
\hline
\end{tabular}

about 1.39\%-3.84\% increase. The learning rate and total loss of Inception-v3 could be found in Figure 3 and respectively. After 40000 iterations, the learning rate and total loss of Inception-v3 began to converge. Then they could remain basically stable.

The specific parameters of the inception-v3 network used in this article were shown in the Table 3 .

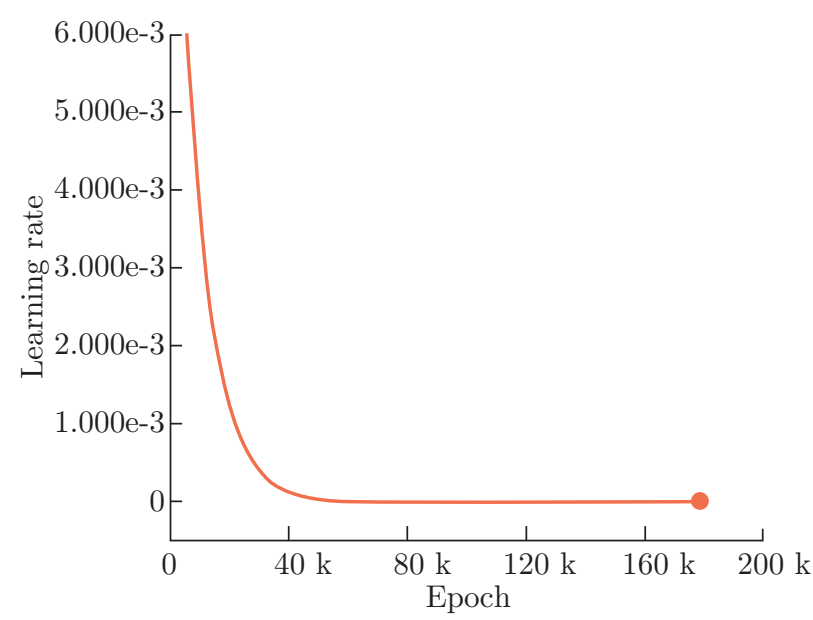

Fig. 3: Learning rate of Inception-v3

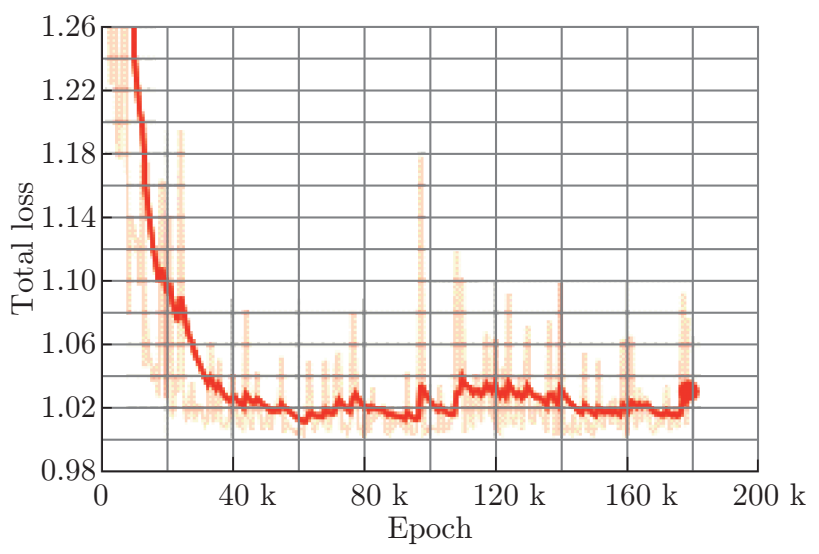

Fig. 4: Total loss of Inception-v3

\section{Conclusion}

This paper presents a method of image classification of female cloth based on deep Convolutional Neural Network. In this paper, a female cloth dataset was built to solve fashion image 
Table 3: Network specific parameters of Inception-v3

\begin{tabular}{ccc}
\hline type & patch size/stride or remarks & input size \\
\hline conv & $3 \times 3 / 2$ & $299 \times 299 \times 3$ \\
conv & $3 \times 3 / 1$ & $149 \times 149 \times 32$ \\
conv padded & $3 \times 3 / 1$ & $147 \times 147 \times 32$ \\
pool & $3 \times 3 / 2$ & $147 \times 147 \times 64$ \\
conv & $3 \times 3 / 1$ & $73 \times 73 \times 64$ \\
conv & $3 \times 3 / 2$ & $71 \times 71 \times 80$ \\
conv & $3 \times 3 / 1$ & $35 \times 35 \times 192$ \\
$3 \times$ inception & As in Figure 5 & $35 \times 35 \times 288$ \\
$5 \times$ inception & As in Figure 6 & $17 \times 17 \times 768$ \\
$2 \times$ inception & As in Figure 7 & $8 \times 8 \times 1280$ \\
pool & $8 \times 8$ & $8 \times 8 \times 2048$ \\
linear & logits & $1 \times 1 \times 2048$ \\
softmax & classifier & $1 \times 1 \times 1000$ \\
\hline
\end{tabular}

classification problem. Three kinds of Convolutional Neural Network are evaluated namely VGG19, Inception-v3, ResNet-v1-50. The results show that 10 categories of female clothing can be correctly classified, the highest accuracy was $96.91 \%$. It turns out that Convolutional Neural Network can efficiently extract features which have good a representation of raw input images. Constructing dataset with more categories can further improve the ability of network model on different fashion image classification task. Subsequent will increase the amount of training data, study more complex deep network structures to train the model, and further improve the accuracy of the classification results. And conduct research based on the classification of clothing attributes.

\section{Acknowledgment}

The authors will thank National Natural Science Foundation for their great help in this paper.

\section{References}

[1] Bao QP, Sun ZF. Clothing Image Classification and Retrieval based on Metric Learning. Computer Applications and Software: 2017, 34(4): 255-259.

[2] Liu S, Feng J, Domokos C, et al. Fashion Parsing with Weak Color-category Labels. IEEE Transactions on Multimedia: 2014, 16(1): 253-265.

[3] Bourdev L, Maji S, Malik J. Describing People: A Poselet-based Approach to Attribute Classification. IEEE Press: 2011, 1543-1550.

[4] Hu Z Yan H, Lin X. Clothing Segmentation Using Foreground and Background Estimation Based on the Constrained Delaunay Triangulation. Pattern Recognition: 2008, 41(5): 1581-1592.

[5] Weber M, Bauml M, Stiefelhagen R. Part-based Clothing Segmentation for Person Retrieval. IEEE Press: 2011, 361-366. 
[6] Manfredi M, Grana C, Calderara S, et al. A Complete System for Garment Segmentation and Color Classification. Machine Vision and Applications: 2014, 25(4): 955-969.

[7] Chen H, Gallagher A, Girod B. Describing Clothing by Semantic Attributes. Springer: 2012, 609-623.

[8] Lorezo-Navarro J, Castrillón M, Ramón E, et al. Evaluation of LBP and HOG Descriptors for Clothing Attribute Description. Springer: 2014, 53-65.

[9] Krizhevsky A, Sutskever I, Hinton G E. Imagenet classification with deep convolutional neural networks. Advances in neural information processing systems: 2012, 1097-1105.

[10] SunY, Liang D, Wang X, et al. Empirical evaluation of rectified activations in convolutional network. arXiv preprint arXiv: 1505.00853, 2015.

[11] Simnyan K, Zisserman A. Two-stream convolutional networks for action recognition in videos. Advances in Neural Information Processing Systems: 2014, 568-576.

[12] Simnyan K, Zisserman A. Very deep convolutional networks for large-scale image recognition. arXiv prepint arXiv: 1409.1556, 2014.

[13] Szegedy C, Liu W, Jia Y, et al. Going deeper with convolutions. Cvpr: 2015, 1-9.

[14] Hinton G E, Salakhutdinov R R. Reducing the dimensionality of data with neural networks. Science: 2006, 313(5786): 504-507.

[15] LaoB, Jagadeesh K. Convolutional Neural Networks for Fashion Classification and Object Detection. http://es231n.stanford: 2015.

[16] Li, Sun YB, Wang F, Liu QS. Clothing Image Classification and Retrieval Algorithm Based on Deep Convolutional Neural Network. Computer Engineering: 2016, 11: 309-315.

[17] Rohi P, Suhas S. Apparel Classification using CNNs. Computer visionaccv: 2012.

[18] Bappy JH, Roy-Chowdhury AK. CNN based region proposals for efficient object detection. 2016 IEEE International Conference on Image Processing (ICIP): (2016)

[19] Chi Z, Li H, Lu H, Yang MH. Dual Deep Network for Visual Tracking. IEEE Trans. Image Process. 26(4): 2005-2015 (2017)

[20] Abadi M, TensorFlow AABP. Large-Scale Machine Learning on Heterogeneous Distributed Systems. Proceedings of the 12th USENIX Symposium on Operating Systems Design and Implementation: 2016, 265-283. 